\title{
Clinical and Economic Outcomes of Rabbit Antithymocyte Globulin Induction in Adults Who Received Kidney Transplants from Living Unrelated Donors and Received Cyclosporine-Based Immunosuppression
}

\author{
James T. Miller, Pharm.D., Curtis D. Collins, Pharm.D., M.S., Linda J. Stuckey, Pharm.D., \\ Fu L. Luan, M.D., Michael J. Englesbe, M.D., John C. Magee, M.D., and Jeong M. Park, Pharm.D. \\ Study Objective. To evaluate the efficacy, safety, and costs of rabbit \\ antithymocyte globulin (TMG) induction in patients who received kidney \\ transplants from living unrelated donors. \\ Design. Retrospective cohort study. \\ Setting. Large academic medical center. \\ Patients. Eighty-seven patients who received kidney transplants from living \\ unrelated donors: 40 of the recipients underwent transplantation between \\ January 1, 2003, and December 31, 2004, and did not receive TMG \\ induction (no induction group); 47 underwent transplantation between \\ January 1, 2005, and June 30, 2006, and received TMG induction (induc- \\ tion group). All patients received cyclosporine-based immunosuppression. \\ Measurements and Main Results. Biopsy-proven acute rejection, \\ posttransplantation complications, and inpatient hospital costs for the first \\ 12 months after transplantation were compared between groups using \\ standard univariate statistical analyses. Induction significantly decreased \\ the occurrence of biopsy-proven acute rejection versus no induction ( $2 \%$ vs \\ $48 \%, p<0.001)$. Fifty percent of rejection episodes in the no induction \\ group required hospitalization, and $46 \%$ of rejection episodes required \\ TMG treatment. Slightly elevated initial costs associated with TMG \\ induction were offset by lower costs related to rejection treatment. Total \\ inpatient costs for the 12 months after transplantation were comparable \\ between the groups (no induction $\$ 66,038$ vs induction $\$ 74,183, \mathrm{p}>0.05$ ). \\ For the no induction versus induction groups, no significant differences in \\ cytomegalovirus disease ( $5 \%$ vs $6 \%$ ), malignancy (3\% vs $2 \%$ ), graft failures \\ ( $5 \%$ vs $6 \%$ ), mortality ( $5 \%$ vs $4 \%$ ), and serum creatinine concentrations \\ (mean \pm SD $1.4 \pm 0.3$ vs $1.5 \pm 0.3 \mathrm{mg} / \mathrm{dl}$ ) were observed at 12 months \\ ( $p>0.05$ for all comparisons). \\ Conclusion. Five-day TMG induction effectively reduced the 1-year acute \\ rejection rate without significantly increasing total inpatient costs or \\ posttransplantation complications among recipients of kidney transplants \\ from living unrelated donors. \\ Key Words: rabbit antithymocyte globulin, Thymoglobulin, living unrelated \\ donor kidney transplantation, rejection, cost. \\ (Pharmacotherapy 2009;29(10):1166-1174)
}

Living-donor kidney transplantation has become a valuable treatment option for end-stage renal disease, and it provides considerable benefits over deceased-donor kidney transplantation. 
These benefits include shorter time on the waiting list and improved graft and patient survival. ${ }^{1,2}$ Living kidney donation is not limited to donor-recipient pairs of biologic linkages. In fact, biologically unrelated donors account for approximately $35 \%$ of living kidney donations in the United States. ${ }^{1}$ The risk of acute rejection was perceived to be lower for living-donor transplant recipients than for deceased-donor transplant recipients, but several studies have provided evidence to the contrary. ${ }^{3-6}$ In particular, living unrelated transplant recipients had higher rejection rates compared with those of deceased-donor recipients and living relateddonor transplant recipients who were given similar maintenance immunosuppression. ${ }^{4-6}$

Acute rejection is a common complication of transplantation that markedly affects long-term clinical and economic outcomes. Patients who experience an episode of acute rejection are more likely to develop chronic rejection and graft failure. ${ }^{7-9}$ This relationship between acute rejection and long-term graft outcome has been shown in all donor types. ${ }^{4,9}$ Acute rejection is also associated with substantial increases in treatment costs and hospitalizations. ${ }^{10,11}$ As an approach to reduce the acute rejection rate, antibody induction is commonly used to intensify initial immunosuppression in kidney transplant recipients.

Several prospective, randomized, controlled clinical trials demonstrated that induction with rabbit-derived polyclonal antithymocyte globulin (Thymoglobulin; Genzyme Corp., Cambridge, MA) reduced the rate and severity of acute rejection compared with no induction or with the use of horse-derived polyclonal antithymocyte globulin or basiliximab. ${ }^{12-15}$ Patients in these studies were primarily recipients of deceaseddonor kidney transplants and included those at

From the Department of Pharmacy Services, Sinai-Grace Hospital, Detroit, Michigan (Dr. Miller); and the Departments of Pharmacy Services (Drs. Collins, Stuckey, and Park), Internal Medicine (Dr. Luan), and General Surgery (Drs. Englesbe and Magee), University of Michigan, Ann Arbor, Michigan.

Supported in part by an investigator-initiated research grant from Genzyme Corporation.

Presented in part at the American College of Clinical Pharmacy annual meeting, Denver, Colorado, October 14-17, 2007.

Manuscript received January 12, 2009. Accepted pending revisions February 23, 2009. Accepted for publication in final form April 23, 2009.

Address reprint requests to Jeong M. Park, M.S., Pharm.D., Department of Pharmacy Services, University of Michigan, UH B2D301/SPC 5008, 1500 East Medical Center Drive, Ann Arbor, MI 48109-5008; e-mail: jeongp@umich.edu. immunologically high risk for rejection; that is, they were generally African-Americans, repeat transplant recipients, patients with high levels of panel reactive antibody, those with a prolonged cold ischemic time, or those with delayed graft function. Although not directly observed in these clinical trials, which had relatively short follow-up periods, a major concern surrounding the use of some induction strategies is that the risk for infections may be increased. Particular issues are cytomegalovirus (CMV) infection, posttransplantation lymphoproliferative disease, and death from infection or malignancy. ${ }^{15-17}$ Because of this safety concern, together with the high acquisition cost of induction agents, many transplant centers limit antibody induction to kidney transplant recipients who are perceived to be at high risk for rejection.

In the past, recipients of kidney transplants from living unrelated donors did not receive antibody induction at our transplant center if they were at low risk for rejection; these included patients who were not of African-American descent, those receiving primary transplants, and those with a panel reactive antibody result below $30 \%$. In 2003, we observed a 1-year acute rejection rate of $57 \%$ among recipients of kidney transplants from living unrelated donors; these transplant recipients had not received antibody induction. To ameliorate the high acute rejection rate, we implemented a TMG-induction protocol for all living unrelated-donor transplant recipients at our transplant center on January 1 , 2005.

The purpose of this study was to evaluate the efficacy, safety, and cost of TMG induction in adults who received kidney transplants from living unrelated donors and who were at immunologically low risk for rejection during the first 12 months after transplantation.

\section{Methods}

\section{Study Design and Patients}

This was a retrospective cohort study approved by our institutional review board. The study population included adults aged 18 years or older who received a primary solitary kidney transplant from a living unrelated donor at our transplantation center between January 1, 2003, and June 30, 2006. Their maintenance immunosuppression consisted of cyclosporine microemulsion, mycophenolate mofetil, and steroids. Patients were excluded if they had received TMG induction for previous transplantation, if they were African- 
American, or if they had a panel reactive antibody result of $30 \%$ or higher.

Eligible patients were divided into a no induction group, which underwent transplantation between January 1, 2003, and December 31, 2004, and a TMG induction group, which underwent transplantation between January 1, 2005, and June 30, 2006. All patients were followed for 12 months after transplantation.

\section{Immunosuppression and Patient Follow-up}

Before 2005, only patients with a previous kidney transplant, those of African-American descent, or those with a panel reactive antibody result of $30 \%$ or greater were considered to have an immunologically high risk for rejection and, therefore, a need for TMG induction. All other low-risk recipients of living unrelated-donor kidney transplants received no antibody induction until January 1, 2005, when we implemented TMG induction for all such patients.

The induction regimen consisted of TMG 1.5 $\mathrm{mg} / \mathrm{kg} /$ day given intravenously for 5 days, with the first dose administered intraoperatively. The dose of TMG was reduced to half for patients with a white blood cell count of 1.5-3.0 x $10^{3} / \mathrm{mm}^{3}$ and withheld for those with a white blood cell count below $1.5 \times 10^{3} / \mathrm{mm}^{3}$ or platelet count below $50 \times 10^{3} / \mathrm{mm}^{3}$.

Both the no induction and induction groups received the same maintenance immunosuppression with cyclosporine microemulsion, mycophenolate mofetil, and steroids. Cyclosporine was started at $5 \mathrm{mg} / \mathrm{kg} /$ dose given orally every 12 hours within 24 hours after surgery. The dosage was adjusted to maintain a morning whole-blood trough concentration of $225-275 \mathrm{ng} / \mathrm{ml}$ for the first month, 175-225 ng/ml for the second month, and $125-175 \mathrm{ng} / \mathrm{ml}$ thereafter. Cyclosporine concentrations were measured centrally by using validated liquid chromatography with tandem mass spectrometry, with an interrun variability of less than 5\%. ${ }^{18}$ Mycophenolate mofetil $1 \mathrm{~g}$ twice/day was started 2 days before transplantation, and the dosage was reduced or temporarily withheld for adverse effects such as gastrointestinal complaints, hematologic abnormalities, or serious infections. Intravenous methylprednisolone $500 \mathrm{mg}$ was given intraoperatively, and oral prednisone was tapered from 100 $\mathrm{mg}$ /day on postoperative day 1 to $5-10 \mathrm{mg}$ /day over 8 weeks.

All patients received CMV prophylaxis with valganciclovir $450 \mathrm{mg} /$ day for 3-6 months, fungal prophylaxis with oral nystatin for 30 days, and Pneumocystis jiroveci prophylaxis with trimethoprim $80 \mathrm{mg}$-sulfamethoxazole $400 \mathrm{mg}$ for 30 days or as a one-time dose of aerosolized pentamidine $300 \mathrm{mg}$.

During the first 12 months after kidney transplantation, patients were followed with a minimum of biweekly laboratory monitoring and weekly-to-quarterly clinic visits, depending on their clinical progress.

All episodes of clinically suspected acute rejection were subsequently confirmed and graded by biopsy according to the Banff 97 criteria. ${ }^{19}$ Once confirmed, borderline or Banff $1 \mathrm{~A}$ acute rejection was treated with intravenous methylprednisolone $250 \mathrm{mg}$ for 3 days, and acute rejection of grade Banff $1 \mathrm{~B}$ or higher was managed with TMG $1.5 \mathrm{mg} / \mathrm{kg} /$ day for $7-14$ days. These treatments were given on an inpatient basis or at our outpatient infusion center, depending on the patient's clinical condition.

\section{Study Assessments}

From the patients' medical records at our hospital, we obtained data regarding recipient and donor characteristics, graft and patient survival, drugs, laboratory data (including cyclosporine trough concentrations, serum creatinine concentrations, and CMV polymerase chain reaction assay results), results of kidney allograft biopsy, and hospitalizations. Costaccounting data were obtained from the hospital's data warehouse. We obtained information for all inpatient resources utilized during hospitalization for the transplantation and for subsequent readmissions to our hospital. Outpatient cost data for drugs, procedures, clinical visits, and hospitalizations to other institutions were not obtained. Costs in the no induction group were discounted to June 2006 dollars. ${ }^{20}$

The primary efficacy end point was the occurrence of biopsy-proven acute rejection in the first 12 months after transplantation. Secondary outcomes were the severity of rejection; occurrence of delayed graft function, graft failure, patient death, CMV disease, and malignancy; patients' renal function at 12 months after transplantation; and number of hospitalizations during the first 12 months. Delayed graft function was defined as a need for dialysis within 1 week after transplantation. Disease due to $\mathrm{CMV}$ was defined as CMV viremia that was detectable with quantitative polymerase chain reaction accompanied by viral syndrome (fever, 
Table 1. Baseline Characteristics

\begin{tabular}{|c|c|c|c|}
\hline Characteristic & $\begin{array}{c}\text { No Induction } \\
\text { Group } \\
(n=40)\end{array}$ & $\begin{array}{c}\text { TMG Induction } \\
\text { Group } \\
(\mathrm{n}=47)\end{array}$ & $\mathrm{p}$ Value \\
\hline \multicolumn{4}{|c|}{ Mean \pm SD } \\
\hline$\overline{\text { Recipient age (yrs) }}$ & $46.3 \pm 11.2$ & $50.3 \pm 12.9$ & 0.13 \\
\hline Donor age (yrs) & $41.0 \pm 9.7$ & $44.7 \pm 11.6$ & 0.11 \\
\hline No. of HLA mismatches & $5.1 \pm 1.2$ & $4.4 \pm 1.0$ & 0.07 \\
\hline HLA-A & $1.5 \pm 0.7$ & $1.2 \pm 0.7$ & 0.21 \\
\hline HLA-B & $1.8 \pm 0.4$ & $1.6 \pm 0.5$ & 0.28 \\
\hline HLA-DR & $1.8 \pm 0.6$ & $1.5 \pm 0.6$ & 0.16 \\
\hline \multicolumn{4}{|c|}{ No. (\%) of Patients } \\
\hline Female recipients & $16(40)$ & $18(38)$ & 0.87 \\
\hline Female donors & $26(65)$ & $29(62)$ & 0.75 \\
\hline Panel reactive antibody result & & & 0.03 \\
\hline $0 \%$ & $36(90)$ & $33(70)$ & \\
\hline $1-9 \%$ & $4(10)$ & $7(15)$ & \\
\hline $10-30 \%$ & $0(0)$ & $7(15)$ & \\
\hline Cause of end-stage renal disease & & & 0.61 \\
\hline Diabetes mellitus & $17(43)$ & $18(38)$ & \\
\hline Hypertension & $4(10)$ & $5(11)$ & \\
\hline Polycystic kidney disease & $8(20)$ & $6(13)$ & \\
\hline Alport's disease & $0(0)$ & $3(6)$ & \\
\hline Obstructive uropathy & $4(10)$ & $3(6)$ & \\
\hline $\begin{array}{l}\text { Focal segmental glomerular } \\
\text { sclerosis }\end{array}$ & $3(8)$ & $4(9)$ & \\
\hline Other & $4(10)$ & $8(17)$ & \\
\hline Cytomegalovirus serostatus & & & 0.93 \\
\hline $\mathrm{D}+/ \mathrm{R}-$ & $10(25)$ & 9 (19) & \\
\hline $\mathrm{D}+\mathrm{R}+$ & $10(25)$ & $12(26)$ & \\
\hline D-/R+ & $9(23)$ & $12(26)$ & \\
\hline D-/R- & $11(28)$ & $14(30)$ & \\
\hline
\end{tabular}

malaise or leukopenia) or organ dysfunc-tion in the absence of other documented causes.

We collected all hospitalization data at our transplant center from the transplantation surgery and during the 12-month follow-up. Hospital readmissions were divided into five categories based on their reason: rejection, infection, surgical complications (urine leak, ureteral obstruction, lymphocele, or wound dehiscence), medical complications (other than infection), and all others.

\section{Statistical Analysis}

Categoric variables were analyzed using a $\chi^{2}$ or the Fisher exact test, and continuous variables were assessed using a Student $t$ test. All tests were two-sided, and statistical significance was defined at a $\mathrm{p}$ value of less than 0.05 .

\section{Results}

Of 161 adults who received living unrelateddonor kidney transplants at our transplant center between January 1, 2003, and June 30, 2006, 87 were included for analysis: 40 patients in the no induction group and 47 patients in the TMG induction group. Baseline characteristics of recipients and donors were similar between the groups, except for the percentage of patients with positive results for panel reactive antibody (Table 1). Also similar between the no induction and induction groups were percentages of donors older than 55 years ( $8 \%$ and $15 \%$, respectively), recipients older than 65 years $(8 \%$ and $6 \%$, respectively), and female donor-to-male recipient pairs (38\% and $43 \%$, respectively; $\mathrm{p}>0.05$ for all comparisons).

Induction of TMG at a mean \pm SD total dose of $5.9 \pm 1.3 \mathrm{mg} / \mathrm{kg}$ was administered with minimal effect on initial hospital stays for transplantation (median days for no induction 3.0 vs induction 4.0). According to our center's protocol, cyclosporine trough concentrations reached target ranges within 7 days in both groups. Mean \pm SD cyclosporine trough concentrations were comparable between the groups at 1 month (no induction $282 \pm 73 \mathrm{ng} / \mathrm{ml}$ vs induction $259 \pm 74$ 
Table 2. Clinical Outcomes at 12 Months After Transplantation

\begin{tabular}{|c|c|c|c|}
\hline Outcome & $\begin{array}{l}\text { No Induction } \\
\text { Group } \\
(\mathrm{n}=38)^{\mathrm{a}}\end{array}$ & $\begin{array}{c}\text { TMG Induction } \\
\text { Group } \\
(\mathrm{n}=44)^{\mathrm{a}}\end{array}$ & $\mathrm{p}$ Value \\
\hline \multirow[t]{2}{*}{$\begin{array}{l}\text { Serum creatinine concentration, } \\
\text { mean } \pm \mathrm{SD}(\mathrm{mg} / \mathrm{dl})\end{array}$} & $1.4 \pm 0.3$ & $1.5 \pm 0.3$ & 0.08 \\
\hline & \multicolumn{2}{|c|}{ No. (\%) of Patients } & \\
\hline $\begin{array}{l}\text { Serum creatinine concentration } \\
>1.5 \mathrm{mg} / \mathrm{dl}\end{array}$ & $10(26)$ & $14(32)$ & 0.59 \\
\hline Acute rejections & $19(50)$ & $1(2)$ & $<0.001$ \\
\hline Severity of first rejection & & & $<0.001$ \\
\hline Borderline & $3(8)$ & $0(0)$ & \\
\hline Banff $1 \mathrm{~A}$ & $5(13)$ & $1(2)$ & \\
\hline Banff 1B & $6(16)$ & $0(0)$ & \\
\hline Banff 2A & $5(13)$ & $0(0)$ & \\
\hline Recurrent rejection & $7(18)$ & $0(0)$ & 0.003 \\
\hline Graft failures & $2(5)$ & $3(7)$ & $>0.99$ \\
\hline Deaths & $2(5)$ & $2(5)$ & 0.63 \\
\hline \multicolumn{4}{|l|}{ Cytomegalovirus disease } \\
\hline Overall & $2(5)$ & $3(7)$ & $>0.99$ \\
\hline $\mathrm{D}+/ \mathrm{R}-$ & $1(3)$ & $3(7)$ & \\
\hline $\mathrm{D}+\mathrm{R}+$ & $1(3)$ & $0(0)$ & \\
\hline $\mathrm{D}-/ \mathrm{R}+$ & $0(0)$ & $0(0)$ & \\
\hline D-/R- & $0(0)$ & $0(0)$ & \\
\hline Malignancies & $1(3)$ & $1(2)$ & 0.71 \\
\hline
\end{tabular}

$\mathrm{ng} / \mathrm{ml}, \mathrm{p}>0.05)$ and throughout the 12 months after transplantation (Figure 1).

Within 12 months after kidney transplantation, 19 patients (48\%) in the no induction group and one patient $(2 \%)$ in the induction group had at least one episode of biopsy-proven acute rejection $(\mathrm{p}<0.001$; Figure 2$)$. Of the 19 patients

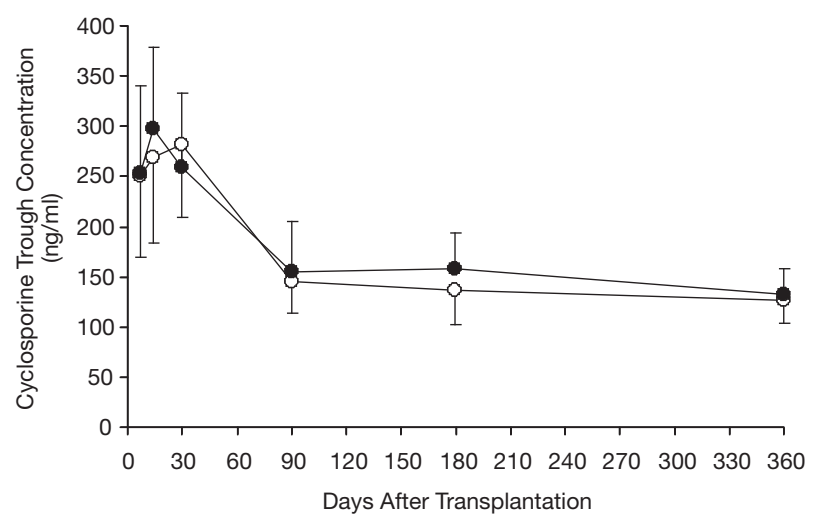

Figure 1. Mean cyclosporine trough concentrations during the 12 months after transplantation for the no induction group (open circles) and the rabbit antithymocyte globulin induction group (closed circles). Error bars indicate SDs. Concentrations were similar at all time points between groups ( $>0.05)$. in the no induction group, 14 (74\%) experienced their first rejection episodes within 30 days after transplantation, seven (37\%) had repeat rejection episodes, and two (11\%) had a third rejection episode. Of 28 rejection episodes in the no

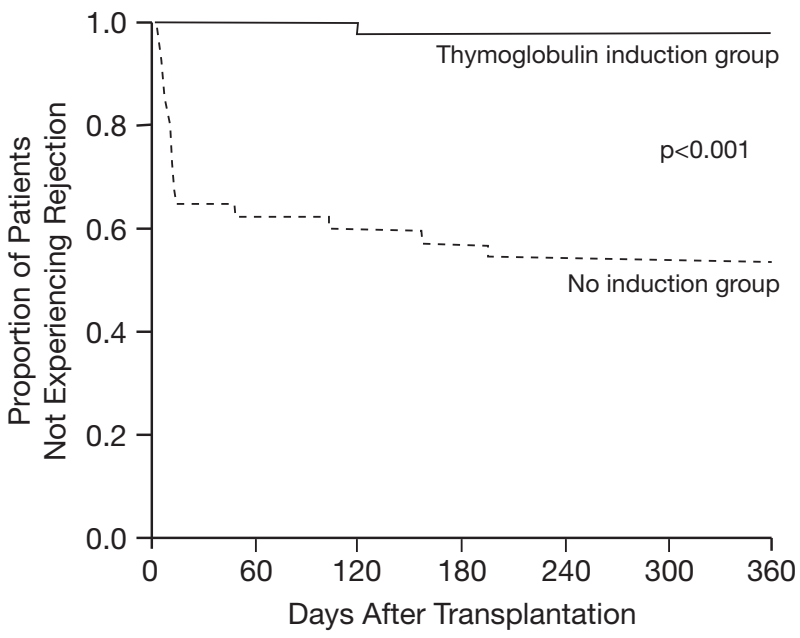

Figure 2. Proportion of patients who did not experience biopsy-proven acute rejection during the 12 months after transplantation in the no induction group and in the rabbit antithymocyte globulin induction group. 
induction group, 13 episodes (46\%) in 12 patients required treatment with TMG (Banff grade $1 \mathrm{~B}$ or higher, $\mathrm{p}<0.001$ vs $\mathrm{TMG}$ induction group), and 14 episodes (50\%) in 13 patients required hospitalization. The one rejection episode (grade Banff 1A) in the induction group was treated with methylprednisolone at the outpatient infusion center (Table 2).

Two deaths occurred in each group. The causes of death were brain herniation and an accident unrelated to transplantation in the no induction group, and hypoglycemia and sepsis in the induction group. Two cases of graft failure occurred in the no induction group, both of which were due to the patient's death. Three cases of graft failure were observed in the induction group; two were due to the patient's death, and one was due to recurrent glomerulonephritis. No case of delayed graft function occurred in either group.

Occurrences of CMV disease and malignancy (squamous cell carcinoma in the no induction group and renal cell carcinoma in the induction group) were low and comparable between the groups. No patients developed BK virus-induced nephropathy or posttransplantation lymphoproliferative disease. Kidney allograft function at 12 months after transplantation, as measured by serum creatinine concentration, was not significantly different between the groups (Table 2). Significantly more kidney allograft biopsy procedures were performed in the no induction group than in the induction group (mean \pm SD $1.1 \pm 1.1$ vs $0.5 \pm 0.7 /$ patient, $\mathrm{p}=0.007)$. This finding indicated that serum creatinine concentrations may have fluctuated more in patients in the no induction group than in patients in the induction group.

During the first 12 months after transplantation, 25 patients in the no induction group had a total of 47 hospital readmissions, 30\% of which were for treatment of rejection episodes. In the induction group, 45 hospital readmissions occurred in 19 patients. Besides rejection, other reasons for hospital readmission were similarly distributed between the groups (Table 3 ).

Hospital resource use is reported in Table 4. Inpatient costs were significantly higher during the hospitalization for transplantation in the induction group than in the no induction group $(\$ 64,722$ vs $\$ 50,628 ; p=0.02)$. However, total hospitalization costs during the first 12 months after transplantation were comparable between the groups (induction $\$ 74,183$ vs no induction $\$ 66,038, p>0.05)$. As expected because of the
Table 3. Hospital Readmissions After Initial Discharge During 12 Months After Transplantation

\begin{tabular}{lcc}
\hline & $\begin{array}{c}\text { No } \\
\text { Induction } \\
\text { Group } \\
(\mathrm{n}=40)\end{array}$ & $\begin{array}{c}\text { TMG } \\
\text { Induction } \\
\text { Group } \\
(\mathrm{n}=47)\end{array}$ \\
\hline Total no. of readmissions & 47 & 45 \\
\hline \multicolumn{3}{c}{ No. (\%) of Readmissions } \\
\hline $\begin{array}{cc}\text { Rejection } \\
\text { Infection }\end{array}$ & $14 / 47(30)$ & $0(0)$ \\
Surgical & $9 / 47(19)$ & $14 / 45(31)$ \\
Medical & $7 / 47(15)$ & $5 / 45(11)$ \\
Other & $14 / 47(30)$ & $19 / 45(42)$ \\
\hline & $3 / 47(6)$ & $3 / 45(7)$ \\
\hline Readmitted & No. (\%) of Patients \\
$\geq 2$ readmissions & $25(63)$ & $19(40)$ \\
\hline & $13(33)$ & $8(17)$ \\
\hline No. of readmissions/patient & $2(1-4)$ & $1(1-6)$ \\
$\begin{array}{l}\text { Duration of hospital stay/ } \\
\text { readmission (days) }\end{array}$ & $5(1-44)$ & $6(1-47)$ \\
\hline TMG = rabbit antithymocyte globulin.
\end{tabular}

Table 4. Costs of Hospital Resource Use During 12 Months After Transplantation

\begin{tabular}{lccc}
\hline & \multicolumn{2}{c}{ Cost/Patient (\$) } & \\
\cline { 2 - 3 } & $\begin{array}{c}\text { No } \\
\text { Induction } \\
\text { Group } \\
(\mathrm{n}=40)\end{array}$ & $\begin{array}{c}\text { TMG } \\
\text { Induction } \\
\text { Group } \\
(\mathrm{n}=47)\end{array}$ & \\
Category & 66,038 & 74,183 & $>0.05$ \\
\hline Total inpatient & & & \\
Transplant & 50,628 & 64,722 & 0.02 \\
hospitalization & 1528 & 6810 & $<0.001$ \\
Pharmacy & 39,137 & 42,773 & $<0.001$ \\
Transplantation & 4546 & 7810 & $>0.05$ \\
Nursing & 5417 & 7329 & $>0.05$ \\
All additional & 15,410 & 9461 & $>0.05$ \\
Readmission & 3985 & 0 & \\
Rejection & 4359 & 3728 & \\
Infection & 1765 & 689 & \\
Surgical & 2841 & 4339 & \\
Medical & 2458 & 705 & \\
All other costs & & & \\
\hline TMG & &
\end{tabular}

TMG = rabbit antithymocyte globulin.

use of TMG, the pharmacy cost/patient was significantly higher in the induction group than in the no induction group during the hospitalization for transplantation (\$6810 vs $\$ 1528$, $\mathrm{p}<0.001)$.

Of note, costs associated with organ procurement and transplanting the donor kidney were \$3636/patient higher in the induction group than in the no induction group. The cost of nursing care tended to be higher with induction, partially due to one outlier in the group who required a 
prolonged stay in the intensive care unit. Although not statistically significant, the mean readmission cost/patient tended to be lower with TMG induction than with no induction (\$9461 vs $\$ 15,410)$.

\section{Discussion}

We present the first comparative data regarding the use of TMG induction versus no induction specifically for immunologically low-risk recipients of living unrelated-donor kidney transplants. In particular, these are patients receiving primary kidney transplants, those not of African-American descent, and those with low panel reactive antibody results. After TMG induction was implemented, the occurrence of acute rejection among recipients of living unrelated-donor kidney transplants dramatically decreased from $48 \%$ to $2 \%$. Most rejection episodes in patients in the no induction group occurred early after transplantation, and about half of the cases required TMG therapy and hospitalization for treatment. The number of patients needed to treat with TMG induction was 2.2 to prevent one episode of acute rejection. This clinical benefit was achieved without significant increases in total inpatient costs or in occurrences of CMV disease and malignancy during the 12 months after transplantation. We observed no apparent differences between the groups in terms of donor and recipient characteristics or maintenance immunosuppression levels that might have suggested that an era effect was responsible for improving outcomes with TMG induction.

The efficacy of TMG induction in our recipients of living unrelated-donor kidney transplants was similar to what was observed in controlled clinical trials of recipients of deceased-donor kidney transplants. ${ }^{12-15}$ Unlike recipients of living related-donor kidney transplants, recipients of living unrelated-donor transplants may be at high risk for rejection partly because of poorer matching of human leukocyte antigens and older donors. ${ }^{5,6}$ However, studies to address proper management of the problem in this specific patient population have been limited to retrospective reviews without comparators.

A retrospective, single-center study revealed an acute rejection rate of $9 \%$ during a mean \pm SD 2.7 \pm 1.7-year follow-up in 68 recipients of living unrelated-donor kidney transplants. ${ }^{21}$ The cohort was given various TMG induction regimens as well as a calcineurin inhibitor, an antiproliferative agent, and steroids. A multicenter registry, Thymoglobulin Antibody Immunosuppression in Living Donor Recipients (TAILOR), involved more than 1500 living-donor kidney transplant recipients treated with TMG induction. ${ }^{22}$ Researchers examining this registry observed a similarly low rejection rate of $6.4 \%$ at 12 months after transplantation, but they did not separately report outcomes for recipients of living unrelated-donor kidney transplants. In both reports, TMG induction was well tolerated, with no serious infectious or malignancy complications observed.

Although our study design was not appropriate to address long-term safety of TMG induction, the short-term (12-mo) outcome was promising, and we observed low and similar occurrences of CMV disease in both patients in the no induction and induction groups who received valganciclovir prophylaxis. In another study, CMV disease was significantly more common in patients who received TMG induction than in those who did not $(32.5 \%$ vs $19.0 \%, \mathrm{p}=0.009){ }^{12}$ However, the authors did not describe whether antiviral prophylaxis was given to their patients. We and others have shown that the potentially increased risk of CMV disease with TMG induction can be overcome by using effective antiviral prophylaxis. ${ }^{21,23,24}$

Another induction approach to reduce acute rejection rates and to potentially improve safety profiles is treatment with interleukin-2 receptor blockers. Published data are lacking about use of this strategy in immunologically low-risk recipients of living unrelated-donor kidney transplants. In deceased-donor kidney transplant recipients, the efficacy of basiliximab induction and calcineurin inhibitor-based triple immunosuppression was clearly inferior to that of TMG induction. ${ }^{12,14}$ In a retrospective analysis of 54 recipients of living related-donor kidney transplants, basiliximab induction failed to show clinical or economic benefits. ${ }^{25}$

The choice of calcineurin inhibitor for maintenance immunosuppression varies across the United States. ${ }^{1}$ Approximately three fourths of kidney transplant recipients in the United States are given tacrolimus, whereas the rest receive cyclosporine microemulsion or no calcineurin inhibitor. Considerable debate surrounds the optimal choice of calcineurin inhibitor. However, a recent randomized clinical trial showed no significant difference between tacrolimus-based regimens and a cyclosporine microemulsion-based regimen. ${ }^{26}$ Also, many 
transplant centers use steroid-sparing immunosuppression regimens. This may limit applicability of our data from living unrelated-donor kidney transplant recipients who received cyclosporine and steroids in addition to mycophenolate mofetil. Conventional triple immunosuppression consisting of tacrolimus, mycophenolate mofetil, and steroids without antibody induction resulted in similar but unacceptably high rejection rates in kidney transplant recipients from both living related donors and living unrelated donors $(57.8 \%$ and $58.8 \%$, respectively, $\mathrm{p}>0.05) .{ }^{27}$ This observation highlights the need for an intensified induction approach in recipients of living unrelated-donor kidney transplants.

As expected, the cost of the primary transplant hospitalization was significantly higher with TMG induction because of the higher pharmacy costs and the increased costs of organ procurement and the transplantation procedure. We theorize that the increased transplantation costs were a function of time. We observed a mean total dose of $5.9 \mathrm{mg} / \mathrm{kg}$ for TMG induction. At this dose, using the average wholesale price, the estimated cost for a $70-\mathrm{kg}$ patient equals $\$ 7434,{ }^{28}$ which can essentially account for the difference in the pharmacy costs between the no induction and TMG induction groups. Of note, the increased costs associated with TMG induction during the primary transplantation hospitalization were eventually offset at 12 months after transplantation by decreased costs due to fewer hospital readmissions associated with rejection.

Whether the increased primary transplantation hospitalization expense is cost-effective depends on several institution-specific factors. First, various doses of TMG for induction have been effective. ${ }^{21,22,29-31}$ We administered TMG $5.9 \pm$ $1.3 \mathrm{mg} / \mathrm{kg}$ over 5 days; however, using a total dose lower than $6 \mathrm{mg} / \mathrm{kg}$ or administering TMG induction over a shorter duration can reduce the cost of the primary transplantation hospitalization. Second, many transplant centers readmit patients for the diagnosis and treatment of rejection. We used outpatient procedures and outpatient infusion centers for this purpose, which could reduce hospital readmission costs.

Retrospective studies based on historical control groups possess inherent limitations. With implementation of the TMG induction protocol at our transplant center, maintenance immunosuppression and other patient management protocols remained the same. Despite this situation, it is possible that there may have been other time-sensitive variables that were not controlled for in our analysis. In addition, this study had a relatively short followup of 12 months and small sample sizes that may have prevented us from detecting potential differences in safety, especially malignancy complications and long-term graft outcomes, between the groups.

Another limitation was the lack of outpatient cost data. Inclusion of outpatient costs for kidney allograft biopsy and rejection treatment at the outpatient procedure and infusion centers could have further increased the total costs for patients in the no induction group. We chose not to include these costs because of the difficulty in obtaining them from the various outpatient sources without bias and because our study was designed from the hospital's perspective. In addition, analyses from the perspective of hospital margins, payer costs, direct and indirect patient costs, and costs to society would be valuable but were beyond the scope of this small analysis. Although TMG induction was associated with higher short-term costs, we hypothesize that the long-term benefits of reduced acute rejection rate and hospital readmissions will offer positive financial returns for the patient, the payer, and society.

A large, prospective, randomized controlled study with a long-term follow-up and complete inpatient and outpatient cost data would be ideal to confirm our hypotheses. However, such studies require substantial resources and are unlikely to be conducted for TMG induction with established efficacy outcomes.

\section{Conclusion}

Five-day TMG induction was an effective and safe strategy to prevent acute rejection in adult recipients of living unrelated-donor kidney transplants. Induction did not significantly increase total inpatient costs during the first year after transplantation. Induction also showed a potential for reducing hospital readmissions and costs associated with the diagnosis and treatment of rejection.

\section{References}

1. U.S. Organ Procurement and Transplantation Network and the Scientific Registry of Transplant Recipients. 2006 OPTN/SRTR annual report: transplant data 1996-2005. Washington, DC: U.S. Department of Health and Human Services, 2006.

2. Matas AJ, Payne WD, Sutherland DE, et al. 2,500 living donor kidney transplants: a single-center experience. Ann Surg 2001;234:149-64. 
3. Campbell SB, Hothersall E, Preston J, et al. Frequency and severity of acute rejection in live- versus cadaveric-donor renal transplants. Transplantation 2003;76:1452-7.

4. Wiland AM, Fink JC, Weir M, et al. Should living-unrelated renal transplant recipients receive antibody induction? Results of a clinical experience trial. Transplantation 2004;77:422-5.

5. Fuller TF, Feng S, Brennan TV, Tomlanovich S, Bostrom A, Freise CE. Increased rejection in living unrelated versus living related kidney transplants does not affect short-term function and survival. Transplantation 2004;78:1030-5.

6. Long DG, Vernon WB, Yanover MJ. Increased acute rejection episodes among recipients of living unrelated donor compared with cadaver and living related donor renal transplants. Transplant Proc 2002;34:1673-4.

7. Almond PS, Matas A, Gillingham K, et al. Risk factors for chronic rejection in renal allograft recipients. Transplantation 1993;55:752-6.

8. Matas AJ, Gillingham K, Humar A, Dunn DL, Sutherland DE, Najarian JS. Immunologic and nonimmunologic factors: different risks for cadaver and living donor transplantation. Transplantation 1999;69:54-8.

9. Knight RJ, Burrows L, Bodian C. The influence of acute rejection on long-term renal allograft survival: a comparison of living and cadaveric donor transplantation. Transplantation 2001;72:69-76.

10. Khan S, Tighiouart H, Kalra A, Raman G, Rohrer RJ, Pereira BJG. Resource utilization among kidney transplant recipients. Kidney Int 2003;64:657-64.

11. Hagenmeyer EG, Häussler B, Hempel E, et al. Resource use and treatment costs after kidney transplantation: impact of demographic factors, comorbidities, and complications. Transplantation 2004;77:1545-50.

12. Mourad G, Garrigue V, Squifflet JP, et al. Induction versus noninduction in renal transplant recipients with tacrolimusbased immunosuppression. Transplantation 2001;72:1050-5.

13. Brennan DC, Flavin K, Lowell JA, et al. A randomized, double-blinded comparison of Thymoglobulin versus Atgam for induction immunosuppressive therapy in adult renal transplant recipients. Transplantation 1999;67:1011-18.

14. Brennan, DC, Daller JA, Lake KD, Cibrik D, Del Castillo D, for the Thymoglobulin Induction Study Group. Rabbit antithymocyte globulin versus basiliximab in renal transplantation. N Engl J Med 2006;355:1967-77.

15. Charpenter B, Rostaing L, Berthoux F, et al. A three-arm study comparing immediate tacrolimus therapy with antithymocyte globulin induction therapy followed by tacrolimus or cyclosporine $A$ in adult renal transplant recipients. Transplantation 2003;75:844-51.

16. Meier-Kriesche HU, Arndorfer JA, Kaplan B. Association of antibody induction with short- and long-term cause-specific mortality in renal transplant recipients. J Am Soc Nephrol 2002;13:769-72.

17. Cherikh WS, Kauffman HM, McBride MA, Maghirang J,
Swinnen LJ, Hanto DW. Association of the type of induction immunosuppression with posttransplant lymphoproliferative disorder, graft survival, and transplantation. Transplantation 2003;76:1289-93.

18. Annesley TM, Clayton L. Simple extraction protocol for analysis of immunosuppressant drugs in whole blood. Clin Chem 2004;50(10):1845-8.

19. Racusen LC, Solez K, Colvin RB, et al. The Banff 97 working classification of renal allograft pathology. Kidney Int 1999;55: 713-23.

20. U.S. Department of Labor, Bureau of Labor Statistics. Consumer price indexes. Available from http://www.bls.gov/cpi. Accessed August 1, 2008.

21. Hardinger KL, Schnitzler MA, Koch MJ, et al. Thymoglobulin induction is safe and effective in live-donor renal transplantation: a single center experience. Transplantation 2006;81:1285-9.

22. Gaber AO, Schnitzler M, Willoughby L, Stirneman P, for the TAILOR Investigators. Thymoglobulin induction in living donor renal transplant recipients: report from TAILOR registry [abstract]. Am J Transplant 2006;6(suppl 2):293.

23. Gelone D, Cibrik D, Volger S, Leichtman A, Lake K. Comparative efficacy and safety of low dose valganciclovir vs. oral ganciclovir for the prevention of cytomegalovirus disease in renal allograft recipients [abstract]. Am J Transplant 2003;3(suppl 5):511.

24. Akalin E, Bromberg JS, Sehgal V, Ames S, Murphy B. Decreased incidence of cytomegalovirus infection in Thymoglobulin-treated transplant patients with 6 months of valganciclovir prophylaxis. Am J Transplant 2004;4:148-9.

25. Crompton JA, Somerville T, Smith L, et al. Lack of economic benefit with basiliximab induction in living related donor adult renal transplant recipients. Pharmacotherapy 2003;23:443-50.

26. Silva HT Jr, Yang HC, Abouljoud M, et al. One-year results with extended-release tacrolimus/MMF, tacrolimus/MMF and cyclosporine/MMF in de novo kidney transplant recipients. Am J Transplant 2007;7(3):595-608.

27. Thorban S, Schwarznau A, Hüser N, Stangl M. Efficacy of conventional immunosuppressive therapy in related and unrelated living renal transplantation. Clin Transplant 2006;20:284-8.

28. Fleming T, ed. Drug topics red book. Montvale, NJ: Thomson PDR, 2006.

29. Agha IA, Rueda J, Alvarez A, et al. Short course induction immunosuppression with Thymoglobulin for renal transplant recipients. Transplantation 2002;73:473-5.

30. Stevens RB, Mercer DF, Grant WJ, et al. Randomized trial of single-dose versus divided-dose rabbit anti-thymocyte globulin induction in renal transplantation: an interim report. Transplantation 2008;85:1391-9.

31. Gurk-Turner C, Airee R, Philosophe D, Kukuruga D, Drachenberg C, Haririan A. Thymoglobulin dose optimization for induction therapy in high risk kidney transplant recipients. Transplantation 2008;85:1425-30. 